\title{
SPECTROPHOTOMETRIC MICRO-ESTIMATION OF BLOOD OXYGEN SATURATION \\ BY
}

\author{
F. E. WEALE* \\ From the Thoracic Surgical Unit, Guy's Hospital \\ Received July 7, 1959
}

Current estimations of oxygen saturation often depend on the measurement of light absorption at two different wavelengths, one being isospectic to hæmoglobin and oxyhæmoglobin, while at the other wavelength there should be a maximal disparity in the light absorption of the two pigments. The formula,

$$
\text { Percentage saturation }=\frac{\left(\frac{E_{a}}{E_{b}}\right)^{H b}-\left(\frac{E_{a}}{E_{b}}\right)^{\text {test }}}{\left(\frac{E_{a}}{E_{b}}\right)^{H b}-\left(\frac{E_{a}}{E_{b}}\right)^{\text {oxy }}} \times 100
$$

where $\mathrm{E}=$ density on a logarithmic scale and $\mathrm{a}=$ any wavelength other than an isospectic $\mathrm{b}$, assumes the theoretical constancy of previously determined absorption ratios for hæmoglobin and oxyhæmoglobin. But King and Wootton (1956) state that these ratios always require checking, even when using the same apparatus from day to day.

The reliability of spectrophotometric methods has been compared with the classical gasometric ones by Wade et al. (1953), and Molyneux and Pask (1955). Holling et al. (1955) conclude that their spectrophotometric method is as accurate as Van Slyke's for all practical purposes.

To justify spectrophotometric methods is therefore no longer necessary. What is needed is the elimination of the errors referred to by King and Wootton, and a simplification of the technique of blood collection and storage without compromising the ideals enunciated by van Slyke (1932). These are the objects of this paper.

The denominators in the four ratios of the above formula should be identical in practice, and it may therefore be rewritten-

$$
\text { Percentage saturation }=\frac{\left(E_{a}\right)^{\mathrm{Hb}}-\left(E_{a}\right)^{\text {test }}}{\left(E_{a}\right)^{\mathrm{Hb}}-\left(E_{a}\right)^{\text {oxy }}} \times 100
$$

Measurement can thus be confined to the wavelength a, as was foreshadowed by Kramer (19.33) and Lemberg and Legge (1949).

\section{APPARATUS}

The Unicam S.P.500 spectrophotometer was used throughout. Square 4-ml. cuvettes provided with it and filled with distilled water were used as standards.

Twenty flat-bottomed test tubes of approximately $1 \frac{1}{2} \mathrm{ml}$. capacity and $8 \mathrm{~mm}$. internal diameter were constricted by means of a spot flame about $8 \mathrm{~mm}$. from their mouths (Fig. 1); this constriction was designed to support a plug of wax, which in turn would support a mercury seal. The tubes are kept on a perspex tray drilled with holes of just the right size to receive them. To fit the tubes into the spectrophotometer detent a special adaptor was made that allowed the light beam to enter the test tube $0.5 \mathrm{~cm}$. from the bottom. The beam, $1 \mathrm{~cm}$. high, was just short of the tube's

* The content of this paper was used in part-fulfilment of the requirements for an M.S. thesis in the University of London. The work was carried out during the tenure of a lectureship in applied physiology at the Royal College of Surgeons. 
shoulder. The level of the beam's entry into the tubes was checked by wrapping slow autopositive paper around them and exposing this in situ. Such centrifuged deposit as might collect at the bottom was shown to have ample room without causing obstruction to the beam. An additional safeguard against optical errors was the placing of a screen, $1 \mathrm{~mm}$. high, in front of the tube's seating, and also the coating of the inside of the adaptor with a non-reflecting black paint. Blood samples were collected in oxalated clean all-glass tuberculin syringes.

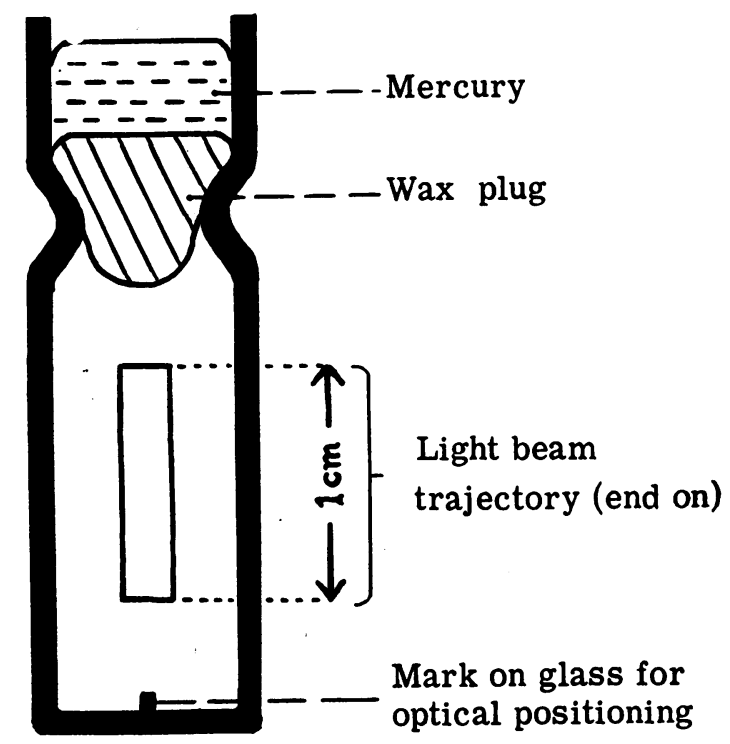

FIG. 1.-Cross-section of test-tube (enlarged).

\section{METHOD}

All tubes are freshly boiled for at least 15 minutes in a beaker of distilled water. Freed from all bubbles but full of water, they are individually extracted with a pair of long forceps. A Pasteur pipette sucks out the water down to the tube constriction, and immediately four drops of molten wax (M.P. $50^{\circ}$ C.) are dropped on to the remaining water. The tube is rapidly cooled in a bath of ice. The resulting contraction of the water draws the wax down, and another drop or two of wax have to be added to fill in the hollow. When the wax has solidified, mercury is added to fill the remaining cup-shaped space at the top (Fig. 1). When all tubes are ready they are stored in a refrigerator until required.

The dead space in the tuberculin syringes is filled with mercury and hub caps are applied to prevent it from falling out. When blood is to be collected through a needle, its dead space is cleared by allowing a small hæmorrhage through it, thus filling it with fresh blood. The syringe is now attached and the plunger withdrawn to the $0.15 \mathrm{ml}$. mark.

The mercury from a sample tube is poured away, a hollow-needle gas jet melts the wax plug, and a tuberculin needle is attached to the sample syringe. Holding the syringe with the hub down so that the mercury blocks its opening, the blood in the hub is discarded. When mercury appears at the tip of the needle, this is plunged through the molten wax and all contents are rapidly expelled. On withdrawal of the syringe, an equivalent volume of water is sucked from the top of the tube so that the wax plug once again solidifies on the tube constriction. Mercury is added as before and, with a finger over its mouth, the tube is quickly inverted to ensure mixing and hæmolysis. It is then replaced in the refrigerator to arrest such oxygen-utilizing metabolism as might still be proceeding.

When all specimens have been collected, they are again shaken to ensure full mixing, and the 
mercury is temporarily poured away while the tubes are centrifuged. They are then ready for reading in the special holder. Using a wavelength of $6650 \AA$, the point of minimum light absorption is found by moving the detent to and fro. When all test readings have been obtained, the wax plugs are scooped out and approximately $0.005 \mathrm{ml}$. of $4 \mathrm{~N}$ ammonia is added to each tube from a tuberculin syringe. Oxygen is bubbled through each tube for a minute, and after centrifugation oxyhæmoglobin readings are taken. Finally $0.02 \mathrm{ml}$. of a saturated watery solution of sodium dithionite is added to each tube and the reduced hæmoglobin values read after centrifuging.

"Internal" checking showed this method to be quite as sensitive as other methods. It has been used routinely on rat blood.

\section{Discussion}

As van Slyke (1932) has pointed out, anything less than a mercury seal for the protection of blood against ambient oxygen contamination is unacceptable. His and other methods of mercury seáling do not surmount the two disadvantages of complicated apparatus for blood collection and ending up with an inverted tube suspended over a mercury well, access to and from which requires some degree of instrumental acrobatics. The glass constriction that holds the wedge of wax which in turn supports the mercury combines the advantages of a mercury seal and easy handling.

The extraction of oxygen from all reagents and apparatus assumes great importance in microestimations. For this reason the tubes are boiled with the water, and potential oxygen carriers such as saponin and bicarbonate are avoided. Saponin indeed may act as deoxygenator (Catton, 1957) and is therefore unsuitable as a hæmolysing agent. Quite apart from the danger of chemical alteration due to it, boiling in order to rid bicarbonate or heparin of their oxygen will variably alter their concentrations; the changing milieu of successive samples will make comparisons more difficult. The boiling of distilled water carries no such disadvantage, and hæmolysis is still adequate.

Other difficulties arise from artifacts inflicted upon the dissociation curve of blood. The traditional addition of alkali avoids turbidity, but it displaces the dissociation curve, particularly of reduced blood, to the left. This will be most noticeable in the "waist" of the curve; the "head" and "tail" are affected much less, and therefore in this method alkali is added only after the first reading has been obtained.

It is an unfortunate property of hæmoglobin that dilution beyond a certain point converts the sigmoid dissociation curve into a right-angled hyperbola. .Experiments with varying dilutions carried out in order to eliminate this cause of falsely high saturation readings showed that dilutions of blood greater than one in ten should be avoided. Wade et al. (1953) did not go to higher dilutions; those of one in a hundred used by Hall (1934) on sheep's blood, as well as introducing this error, might also lead to euglobulin precipitation. If one aims at dilutions higher than one in ten, the addition of the heat-labile substances that normally diminish oxygen avidity of hæmoglobin within the erythrocyte (Lemberg and Legge, 1949) might provide the key.

\section{SUMMARY}

A spectrophotometric method for the measurement of hæmoglobin oxygen saturation is presented.

Measurements at one wavelength only avoid errors due to changing absorption ratios.

Measurement carried out in one and the same vessel throughout eliminates the need for accurate optical matching and accurate volumetric control of test solutions.

A new kind of mercury seal for the preservation of blood samples is described.

My thanks are due to Dr. Cyril Long for much help.

\section{REFERENCES}

Catton, W. T. (1957). Physical Methods in Physiology. Pitman and Sons, Ltd., London.

Hall, F. G. (1934). J. Physiol., 80, 502.

Holling, H. E., MacDonald, I., O'Halloran, J. A., Venner, A. (1955). J. appl. Physiol., 8, 249. 
King, E. J., Wootton, I. D. P. (1956) Microanalysis in Medical Biochemistry. J. and A. Churchill, London. Kramer, K. (1933). Klin. Wchschr., 12, 1875.

Lemberg, R., Legge, J. W. (1949). Hematin Compounds and Bile Pigments. Interscience Publishers Ltd., London and New York.

Molyneux, L., Pask, E. (1955). Brit. Heart J., 17, 169.

Van Slyke D. D. (1932). in Vol. 2 of Quantitative Clinical Chemistry. Williams and Wilkins, Baltimore.

Wade, O. L., Bishop, J. M., Cumming, G., Donald, K. W. (1953). Brit. med. J., 2, 902. 